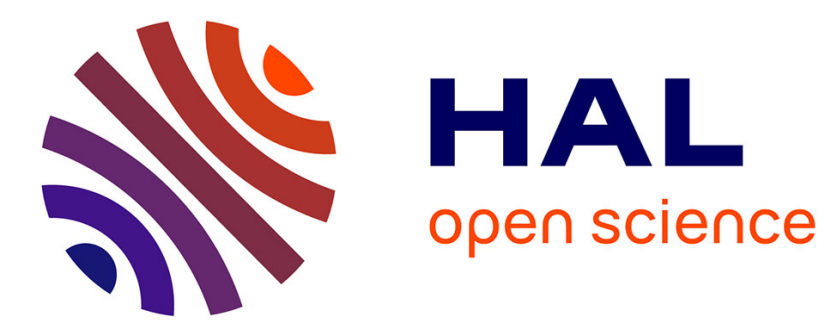

\title{
Wavelet dimensions and time evolution
}

Charles-Antoine Guérin, Matthias Holschneider

\section{To cite this version:}

Charles-Antoine Guérin, Matthias Holschneider. Wavelet dimensions and time evolution. J. C. van den Berg Wavelets in Physics, Cambridge University Press, pp.421-448, 1999, 9780521593113. 10.1017/CBO9780511613265.014 . hal-00083218

\section{HAL Id: hal-00083218 https://hal.science/hal-00083218}

Submitted on 13 Jul 2016

HAL is a multi-disciplinary open access archive for the deposit and dissemination of scientific research documents, whether they are published or not. The documents may come from teaching and research institutions in France or abroad, or from public or private research centers.
L'archive ouverte pluridisciplinaire HAL, est destinée au dépôt et à la diffusion de documents scientifiques de niveau recherche, publiés ou non, émanant des établissements d'enseignement et de recherche français ou étrangers, des laboratoires publics ou privés. 


\title{
11 \\ Wavelet dimensions and Time evolution
}

\author{
Charles-Antoine Guérin, \\ Centre de Physique Théorique, Marseille \\ and \\ Matthias Holschneider, \\ Centre de Physique Théorique, Marseille
}

\begin{abstract}
In this chapter, we study some aspects of the chaotic behaviour of the time evolution generated by hamiltonian systems, or more generally dynamical systems. We introduce a characteristic quantity, namely the lacunarity dimension, to quantify the intermittency phenomena that can arise in the time evolution. We then focus on the time evolution of wave packets according to the Schrödinger equation with time independent hamiltonian. We introduce a set of fractal dimensions constructed by means of the wavelet transform, the (generalized) wavelet dimensions. We show that the lacunarity dimension of the wave packets can be obtained via the wavelet dimensions of the spectral measure of the Schrödinger operator. This establishes a precise link between the long time chaotic behaviour of the wave packets and the small scales spectral properties of the hamiltonian.
\end{abstract}




\subsection{Introduction}

In this chapter, we are interested in the characterization of some intermittency phenomena that can arise in chaotic dynamical systems. Our aim is to introduce parameters to quantify the strength of intermittency in a turbulent signal. To motivate the discussion, let us begin with a simple example. Consider a particle whose motion in $X \subset \mathbb{R}^{n}$ is governed by some hamiltonian system

$$
\begin{aligned}
& \frac{\partial q}{\partial t}=\frac{\partial H}{\partial p} \\
& \frac{\partial p}{\partial t}=-\frac{\partial H}{\partial q}
\end{aligned}
$$

where $q(t) \in \mathbb{R}^{n}$ and $q(t) \in \mathbb{R}^{n}$ are the conjugate generalized coordinates at time $t$. Denote by $T^{*}(X) \subset \mathbb{R}^{2 n}$ the phase space associated to the motion and $x(t)=(q(t), p(t))$ the position of the particle in phase space. If the Hamiltonian $H$ is time independent, the evolution of $x(t)$ is given by a flow $\Phi_{t}$, that is a one parameter semi group of transformations

$$
x(t+s)=\Phi_{t}(x(s)), t, s \geq 0 .
$$

By Liouville's theorem, the area in phase space is conserved under the hamiltonian flow. Precisely, we have for any bounded region $A$ in $T^{*}(X)$ :

$$
\int_{A} d p d q=\int_{\Phi_{t} A} d p d q
$$

Thus the "surface" measure (this is actually a surface for $n=1$ ) on the phase space

$$
\mu(A)=\int_{A} d p d q
$$

is invariant under $\Phi_{t}$. Furthermore, if the phase space $T^{*}(X)$ is compact, then $\mu$ is finite.

Now suppose we can evaluate the location of the particle in phase space periodically in time (with some period say $\tau$ ) by means of some stroboscopic system, that is we are given a discrete set of values $x_{n}=$ $x(n \tau)$. The passage from $x_{n}$ to $x_{n+1}$ reads

$$
x_{n+1}=F\left(x_{n}\right),
$$

where $F=\Phi_{\tau}$ is the evolution operator over one period. Thus the system $\left(T^{*}(X), \mu, F\right)$ is a discrete dynamical system associated to the 


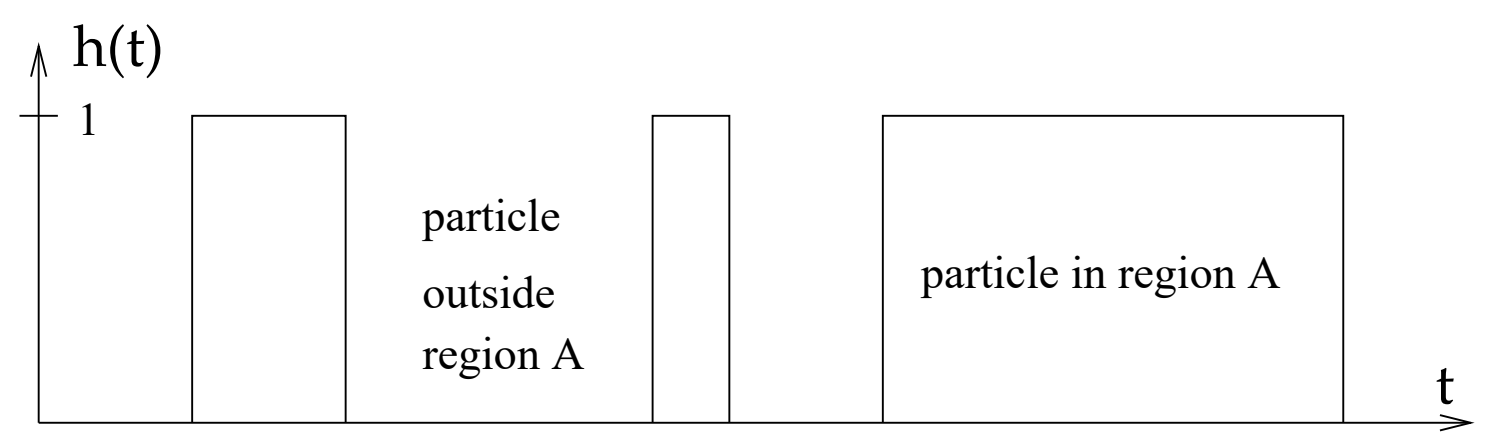

Fig. 11.1. Theoretical characteristic function of the motion.

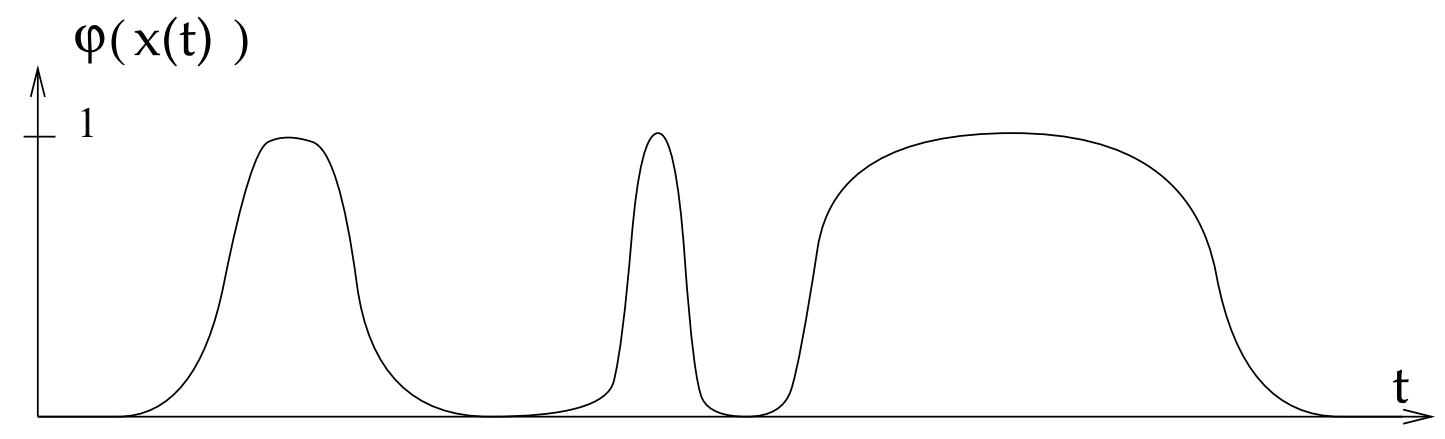

Fig. 11.2. Observed characteristic function of the motion.

finite invariant measure $\mu$. It follows from the Poincaré recurrence theorem that $\mu$-almost every point of a region in phase space is recurrent. Precisely, for all $A \subset T^{*}(X)$, there is a set $B \subset A$ with $\mu(B)=\mu(A)$ such that for all $x_{0} \in B$, the sequence $\left(x_{n+1}\right)$ returns infinitely many times in $A$.

Now a natural question arises. How frequently does the particle return to the same region $A$ of phase space ? This can be visualized by forming the function

$$
h(t)=\chi_{A}(x(t)),
$$

where $\chi_{A}$ is the characteristic function of $A$

$$
\chi_{A}(x)=\left\{\begin{array}{l}
1 \text { if } \mathrm{x} \in \mathrm{A} \\
0 \text { elsewhere. }
\end{array}\right.
$$

The recurrent motion of the particle is mirrored in the intermittent behaviour of $h(t)$ (figure 11.1). The more lacunary this function is, the sparser is the come back in region $A$. Thus, the strength of intermittency is characterized by the degree of lacunarity of the $h(t)$.

Now let us state the problem in a more abstract and general framework. Consider a particle whose motion $x(t)$ in some phase space, possi- 
bly unbounded, is given by an arbitrary dynamical system and as before test if the particle is present or not in some fixed region $A$ by looking at the function $h(t)=\chi_{A}(x(t))$. The physical windowing system which corresponds to the characteristic function may not be perfect, so it is more natural to take $h(t)=\varphi(x(t))$, where $\varphi$ is some smooth positive function well localized in region $A$ (figure 11.2).

At instant $T$, the fraction of time $\langle h\rangle_{T}$ spent by the particle in region $A$ is

$$
<h>_{T}=\frac{1}{T} \int_{0}^{T} d t h(t) .
$$

If $\langle h\rangle_{T}$ converges toward some finite constant as $T \rightarrow \infty$, the limit can be interpreted as a rate of presence in region $A$. If the particle never returns in $A$, then $\langle h\rangle_{T} \sim T^{-1}, T \rightarrow \infty$. In the general case where the particle returns intermittently in $A$, we may expect some overall decrease of the form $\langle h\rangle_{T} \sim T^{-\alpha}, T \rightarrow \infty$. The scaling may or may not exist. However, we can always define the following exponents

$$
d_{0}^{+}[h]=\limsup _{T \rightarrow \infty} \frac{\log \left(\int_{0}^{T} d t h(t)\right)}{\log T}, d_{0}^{-}[h]=\liminf _{T \rightarrow \infty} \frac{\log \left(\int_{0}^{T} d t h(t)\right)}{\log T} .
$$

The problem is that $\langle h\rangle_{T}$ is an average quantity and therefore only gives a rough idea of the real time evolution. Indeed, for given exponents $d_{0}^{ \pm}[h]$ several scenarios are possible. For instance, think of a particle going further and further away from its initial localization in phase space so that $\langle h\rangle_{T} \sim T^{-1}$ as $T \rightarrow \infty$ and therefore $d_{0}^{+}[h]=d_{0}^{-}[h]=0$. Another situation is a particle wandering somewhere in phase space but returning infinitely many times in the same region $A$ with more and more time needed for each come back in such a way that the fraction of time spent in $A$ still scales like $T^{-1}$ whence again $d_{0}^{+}[h]=d_{0}^{-}[h]=0$.

Thus, it appears that the exponents $d_{0}^{+}$and $d_{0}^{-}$are not capable to detect the intermittent nature of the motion. To get a sharper description, we propose to consider not only the mean value $\langle h\rangle_{T}$ but also the higher momenta

$$
<t^{m} h>_{T}=\frac{1}{T^{m+1}} \int_{0}^{T} d t t^{m} h(t), m=1,2 \ldots
$$

and the associated upper and lower exponents

$$
d_{m}^{+}[h]=\limsup _{T \rightarrow \infty} \frac{\log \left(\int_{0}^{T} d t t^{m} h(t)\right)}{\log T}, d_{m}^{-}[h]=\liminf _{T \rightarrow \infty} \frac{\log \left(\int_{0}^{T} d t t^{m} h(t)\right)}{\log T} .
$$


Note that the above exponents are invariant under a time translation $h(t) \rightarrow h\left(t+t_{0}\right)$, that is the time origin that we have taken to be 0 can actually be any arbitrary constant. In the next section, we will prove that the limit

$$
d_{l a c}[h]=\lim _{m \rightarrow \infty} \frac{d_{m}^{-}[h]}{m}
$$

exists. We will call it lacunarity dimension because it measures, in some sense, the degree of lacunarity of a positive function. Then, we will focus on a case of quantum chaos and show that the lacunary character of the time evolution can be related to fractal spectral properties of the corresponding Hamiltonian via the fractal wavelet dimensions .

\subsection{The lacunarity dimension}

Since the above definition of the lacunarity dimension is not at all intuitive, let us motivate it by looking at the following simple example.

Example 1 Consider the function:

$$
h(t)=\sum_{n=0}^{\infty} \delta\left(t-b_{n}\right),
$$

where $\delta(t)$ is the Dirac function

$$
\delta(t)= \begin{cases}1 & \text { if } \mathrm{t}=0 \\ 0 & \text { else }\end{cases}
$$

This can be seen as the characteristic function of a motion with infinitely short times of sojourn in some region of recurrence, the $b_{n}$ corresponding to the successive instants of return. Here, we choose a sequence $\left(b_{n}\right)$ which becomes more and more lacunary as $n$ increases, precisely

$$
b_{n+1} \sim b_{n}^{\gamma}, n \rightarrow \infty
$$

with $\gamma>1$ and $b_{0}>1$. In this case, $d_{m}^{+}[h]$ and $d_{m}^{-}[h]$ can be computed explicitly. Indeed we have, for all $T \geq b_{0}$,

$$
\int_{0}^{T} d t t^{m} h(t)=\sum_{b_{n} \leq T} b_{n}^{m} \sim b_{N}^{m}, T \rightarrow \infty,
$$

where $N$ is the unique integer such that $b_{N} \leq T<b_{N+1}$. The log-log 


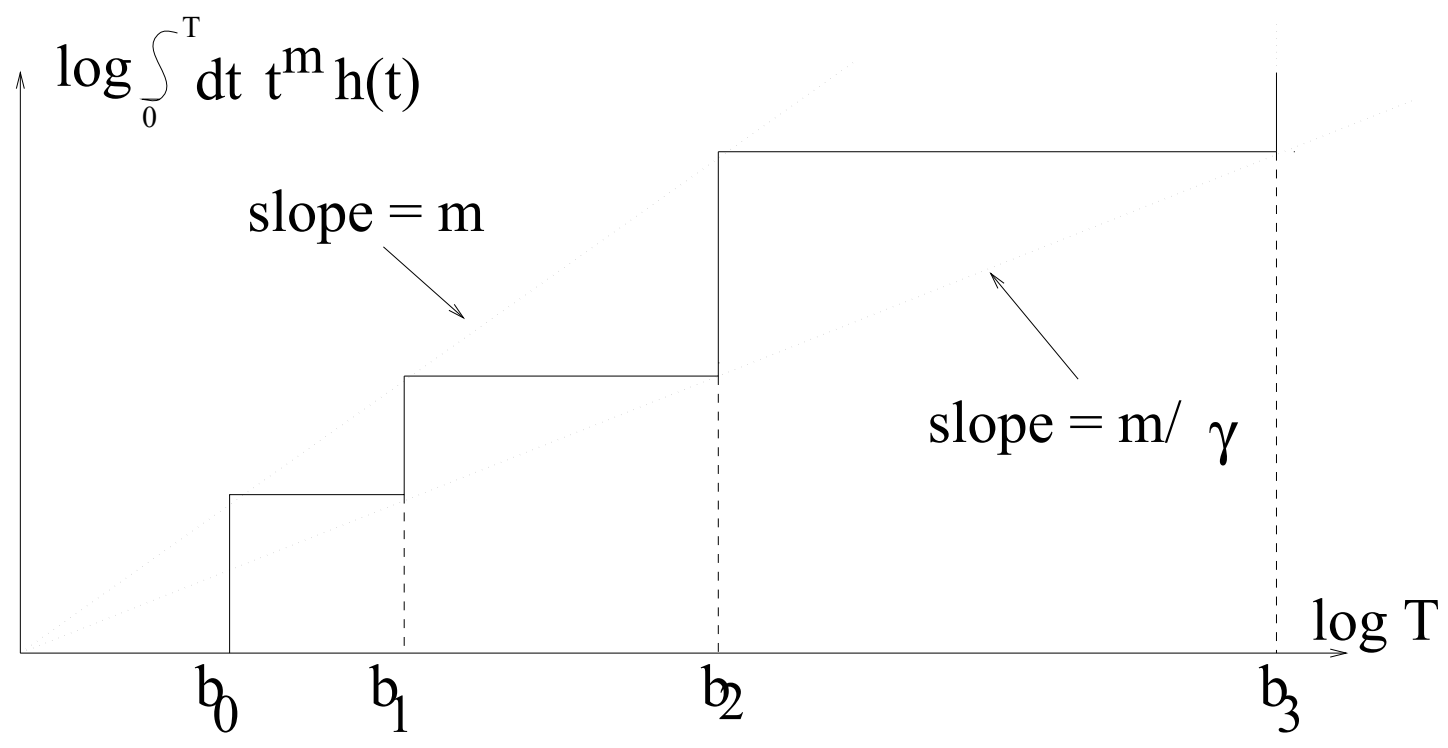

Fig. 11.3. Computation of $d_{m}^{ \pm}[h]$ in a simple case.

diagram of the function $\int_{0}^{T} d t t^{m} h(t)$ is plotted on figure 11.3. Clearly, it appears that:

$$
d_{m}^{+}[h]=\lim _{N \rightarrow \infty} \frac{\log \left(\int_{0}^{b_{N}} d t t^{m} h(t)\right)}{\log b_{N}}=m,
$$

and

$$
d_{m}^{-}[h]=\lim _{N \rightarrow \infty} \frac{\log \left(\int_{0}^{b_{N}} d t t^{m} h(t)\right)}{\log b_{N+1}}=\frac{m}{\gamma},
$$

that is the upper and lower exponents $d_{m}^{+}[h]$ and $d_{m}^{-}[h]$ have different rates of growth in $m$. Now this example supplies motivation for the following

Theorem 11.2.1 Let $h$ be a positive measurable function such that $d_{0}^{+}[h]<\infty$. Then the limit

$$
d_{l a c}[h]=\lim _{m \rightarrow+\infty} \frac{d_{m}^{-}[h]}{m}
$$

exists and satisfies $0 \leq d_{l a c}[h] \leq 1$. Moreover, the limit

$$
\lim _{m \rightarrow+\infty} \frac{d_{m}^{+}[h]}{m}
$$

also exists and is trivial in the sense that it is either 0 or 1 . We call $d_{l a c}[h]$ the lacunarity dimension of $h$ and we say the function $h$ is lacunary if $d_{l a c}<1$. 
polynomial

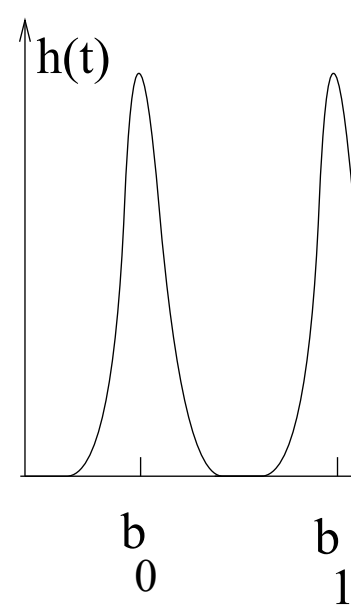

rate of growth

of the gaps localization

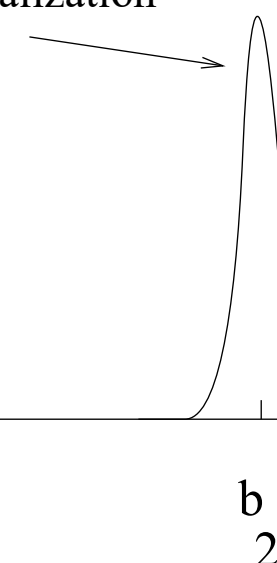

b

Fig. 11.4. Lacunary function

Since the proof is quite heavy, although not difficult, we have defered it to the appendix.

The example given above to introduce the lacunarity dimension is instructive but not realistic because the true characteristic function of a motion cannot be expressed in terms of Dirac functions (the speed of the particle is finite!). Therefore, the example needs to be refined by taking account of the time of sojourn in the region of recurrence. We now consider the following

Example 2 Let $h(t)$ be a positive function which can be written as a superposition of polynomially localized bumps centered a instants $b_{n}$

$$
h(t)=\sum_{n=0}^{\infty} \varphi\left(t-b_{n}\right),
$$

where

$$
\varphi(t)=(1+|t|)^{-K}
$$

We take $K>1$ and again we assume the $b_{n}$ to scale asymptotically like $b_{n+1} \sim b_{n}^{\gamma}, n \rightarrow \infty$, with $\gamma>1$ and $b_{0}>1$. Such a function is illustrated in figure 11.4. Straightforward computations leads to the following expressions for $d_{m}^{+}[h]$ and $d_{m}^{-}[h]$. If $m-K+1 \leq m / \gamma$, as can occur for small $m$, then

$$
d_{m}^{+}[h]=m, \text { and } d_{m}^{-}[h]=\frac{m}{\gamma},
$$


else if $m / \gamma \leq m-K+1$ (for large $m$ ), then

$$
d_{m}^{+}[h]=m, \text { and } d_{m}^{-}[h]=m-K+1 .
$$

If the function $\varphi$ is exponentially localized, $\varphi(t)=e^{-|\sigma t|}$, we obtain $d_{m}^{+}[h]=m$ and $d_{m}^{-}[h]=\frac{m}{\gamma}$ for all $m$. The proof is given in the appendix.

Again, we see that the introduction of a weight $t^{m}$ in the averages tends to separate the upper and lower exponents $d_{m}^{+}[h]$ and $d_{m}^{-}[h]$, at least for the lowest momenta, and thus makes the lacunarity more visible. Note that here $d_{0}^{+}[h]=d_{0}^{-}[h]=0$. Therefore the classical averages $\int_{0}^{T} d t h(t)$ do not reveal the chaotic behaviour of the function $h$, whereas the higher momenta do. Indeed, the rate of growth of $d_{m}^{-}[h]$ as $m$ increases in the first regime ( small $m$ ) gives access to $\gamma$. This parameter tells how fast the gaps enlarge with the time, that is it quantifies the strength of intermittency in the time evolution. The value of $m$ for which the regime transition occurs give access to the parameter $K$, which measures the accuracy of the bumps, that is the form of the window $\varphi$. In this example, we have a competition between the lacunarity of the sequence $\left(b_{n}\right)$ and the localization of the function $\varphi(t)$. When $m$ increases, the bumps $t^{m} \varphi(t)$ become less amd less well separated and so the lacunarity becomes less and less apparent. This explains why for large $m$ the exponent $d_{m}^{-}[h]$ does not depend anymore of the parameter $\gamma$ if $\varphi$ is only polymomially localized. In that case, we have actually $d_{l a c}[h]=1$ and thus the lacunary behaviour of $h(t)$ is not shown up with our definition. However, we can observe $d_{m}^{-}[h] \propto m / \gamma$ on some range (see figure 11.5), from which we deduce that $h$ is lacunary but with a bad localization. Note that the same kind of problem often arises with fractal dimensions in physics. Some natural objects can be assimilated to fractals up to a certain scale, but the fractality breaks down when one looks at too small a scale. For these objects, the fractal dimension with a theorical definition is trivial although a certain scaling law exists in some range of scales.

Now let us make some comments on the choice of the sequence $b_{n}$. In the above example, we took the instants of return $b_{n}$ to grow like $b_{n+1} \sim$ $b_{n}^{\gamma}, \gamma>1$, and with this assumption we obtained $d_{l a c}[h]=1 / \gamma$ ( at least for exponentially localized window function $\varphi$ ). This example can appear somewhat artificial and restrictive. However, in many cases, one can boil down to this kind of lacunary functions by a simple change of variables. For instance, if the $b_{n}$ grow in a geometrical ratio, $b_{n+1} \sim \gamma b_{n}, n \rightarrow \infty$, 


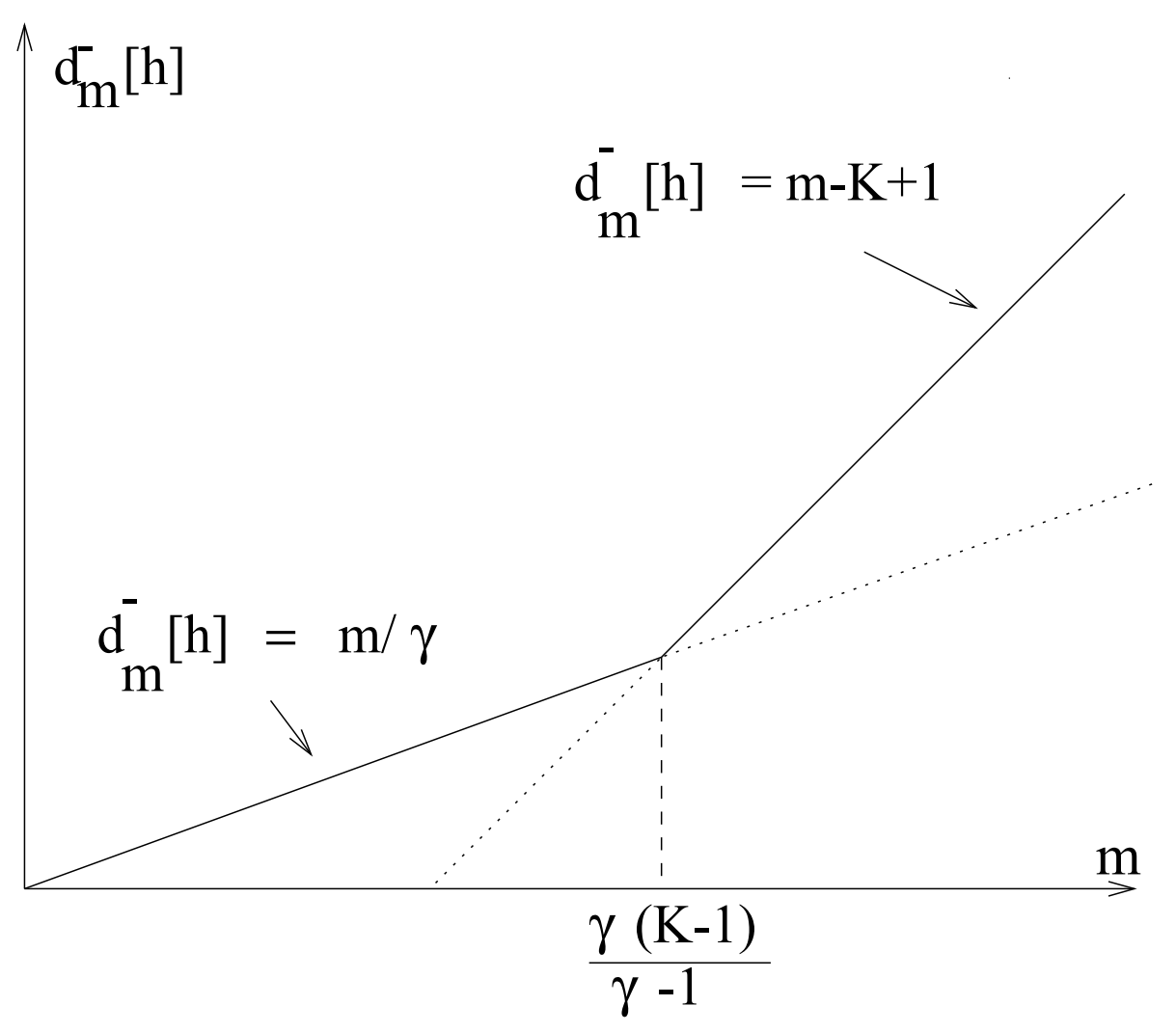

Fig. 11.5. Different regimes for $d_{m}^{-}[h]$

then it is not hard to verify that for exponentially localized bumps we have $d_{l a c}[h]=1$ but $d_{l a c}[h \circ \log ]=1 / \gamma$, that is $h(\log t)$ is lacunary.

We end this section with a negative result which allows us to restrict the set of lacunary functions.

Proposition 11.2.1 Let $h(t)$ be a positive measurable function. If for some $m_{0} \geq 0$ we have

$$
d_{m_{0}}^{+}[h]=d_{m_{0}}^{-}[h]=\alpha>0,
$$

then $d_{l a c}[h]=1$, that is $h$ cannot be lacunary.

The proof is given in the appendix. This statement in particular excludes all the functions $h(t)$ satisfying $\langle h\rangle_{T} \sim T^{-D}$ with $0<D<1$ to be lacunary, because in that case $d_{0}^{+}[h]=d_{0}^{-}[h]=1-D>0$.

\subsection{Quantum chaos}

We will now study the problem of intermittent time evolution in the framework of quantum mechanics. Consider a particle whose motion is 
now governed by the Schrödinger equation

$$
\frac{\partial \psi_{t}}{\partial t}=-i H \psi_{t}
$$

where $\psi_{t} \in L^{2}\left(\mathbb{R}^{n}\right)$ is the wave function of the particle at time $t$. The Hamiltonian $H$ is a self adjoint operator acting on the Hilbert space $\mathcal{H}=L^{2}\left(\mathbb{R}^{n}\right)$. If $H$ is time independent, the dynamics of this system is given by the evolution operator $e^{-i H t}$

$$
\psi_{t}=e^{-i H t} \psi_{0}
$$

The evolving state $\psi_{t}$ usually spreads in configuration space and looses its initial localization. This spreading is estimated by the so called survival probability $\left|\left\langle\psi_{t} \mid \psi_{0}\right\rangle\right|^{2}$. More generally, the space time behaviour of the wave packets can be estimated by comparing $\psi_{t}$ with some reference state $\phi$ in $\mathcal{H}$. Let us define

$$
h(t)=\left|\left\langle\psi_{t} \mid \phi\right\rangle\right|^{2} .
$$

This quantity is the probability for the state $\psi_{t}$ to be in configuration $\phi$ or more simply, if $\phi$ is the characteristic function of some region $\Omega \subset \mathbb{R}^{n}$, this is the probability of finding the particle in region $\Omega$ at time $t$. Now let us introduce $\mu$ : the spectral measure of $H$ associated to $\psi_{0}$ and $\phi$, uniquely defined by (see e.g [6])

$$
<f(H) \psi_{0}, \phi>=\int d \mu(x) f(x)
$$

for all measurable functions $f$. From (11.3.1) and (11.3.2) it follows that

$$
h(t)=|\widehat{\mu}(t)|^{2},
$$

where $\widehat{\mu}$ is the Fourier transform of $\mu$

$$
\widehat{\mu}(t)=\int d \mu(x) e^{-i t x} .
$$

Thus, the evolution of $\psi_{t}$ is governed by the Fourier transform of the spectral measure. It is therefore natural to try to relate the long time behaviour of $h(t)$ to the spectral properties of the hamiltonian. So some heuristic arguments have been given in [4] supporting the fact that the averages

$$
<h>_{T}=\frac{1}{T} \int_{0}^{T} d t h(t) .
$$

exhibit a scaling behavior $\langle h\rangle_{T} \sim T^{-D}$ where $D$ is a fractal dimension 
of the measure $\mu$, namely the correlation dimension (e.g [5]). In [2] some new fractal dimensions have been introduced by means of wavelet transforms, namely the $q$-wavelet dimensions $\kappa_{q}^{ \pm}, q=1,2 \ldots$ For these dimensions it has been shown that the heuristic argument is actually true and that the long time evolution of $\langle h\rangle_{T}$ is governed by the upper respectively lower 2-wavelet dimension $\kappa_{2}^{+}[\mu]$ and $\kappa_{2}^{-}[\mu]$, also called upper and lower wavelet correlation dimension. Precisely we have $d_{0}^{+}[h]=-\kappa_{2}^{-}[\mu]$ and $d_{0}^{-}[h]=-\kappa_{2}^{+}[\mu]$. In the following, we want to show that an easy generalization of these to the $q$-wavelet dimensions makes it possible to express the exponents $d_{m}^{ \pm}[h]$, and consequently the lacunarity dimension $d_{l a c}[h]$, in terms of fractal dimensions of $\mu$. In the next section, we introduce our main tool, the wavelet transform. Then we define a two parameter set of wavelet dimensions $\kappa_{q, m}^{ \pm}$, which we relate to the exponents $d_{m}^{ \pm}[h]$ In order not to get to far off the main flow of argument, the long or technical proofs have been relegated to the appendix.

\subsection{The generalized wavelet dimensions.}

We now wish to introduce the wavelet dimensions. We will first make some brief recall on the wavelet analysis and list a few properties that are necessary for the following. We follow here the notations of [3]. A wavelet is basically a complex valued function $g$ of zero mean $\left(\int g=0\right)$, which is well localized both in real space and Fourier space (this will soon be made more precise). The wavelet transform of a complex valued function $s$ with respect to an analysing wavelet $g$ is given by

$$
\mathcal{W}_{g} s(b, a)=\int d t \frac{1}{a} \bar{g}\left(\frac{t-b}{a}\right) s(t) .
$$

or in Fourier space

$$
\mathcal{W}_{g} s(b, a)=\frac{1}{2 \pi} \int d \omega e^{i \omega b} \widehat{\widehat{g}}(a \omega) \widehat{s}(\omega)
$$

where $\wedge$ is the usual Fourier transform on $\mathcal{S}(\mathbb{R})$

$$
\widehat{g}(\omega)=\int d x e^{-i \omega x} g(x) .
$$

This is a function over the position-scale half plane $\mathbb{I H}=\mathbb{R} \times \mathbb{R}^{+}$. Intuitively, the wavelet transform acts as a filter selecting the details 
present in $s$ at scale $a$ and position $b$. If we introduce the following notations, to be maintained in the remainder

$$
g_{b, a}(t)=\frac{1}{a} g\left(\frac{t-b}{a}\right), g_{a}(t)=\frac{1}{a} g\left(\frac{t}{a}\right), \tilde{g}(t)=\bar{g}(-t),
$$

then the wavelet transform may be seen as a convolution

$$
\mathcal{W}_{g} s(b, a)=\int d t \bar{g}_{a}(t-b) s(t)=\tilde{g}_{a} * s(b)
$$

or a family of scalar products in $\mathbb{R}$

$$
\mathcal{W}_{g} s(b, a)=\int d t \bar{g}_{b, a}(t) s(t)=\left\langle g_{b, a} \mid s\right\rangle .
$$

Thus, the wavelet analysis consists in comparing some function to a family of dilated and translated versions $g_{b, a}$ of a mother wavelet $g$. The wavelet synthesis of a function $\mathcal{T}$ over $\mathbb{H}$ with respect to a reconstructing wavelet $h$ is given by

$$
\mathcal{M}_{h} \mathcal{T}(t)=\int_{H} \frac{d a}{a} d b \mathcal{T}(b, a) \frac{1}{a} h\left(\frac{t-b}{a}\right) .
$$

This is essentially the inverse of the wavelet transform. Now let us introduce the function spaces on which the wavelet analysis is to be developed. Let $\mathcal{S}(\mathbb{R})$ be the Schwartz space of $C^{\infty}$ functions $\varphi$ which, together with their derivatives, are rapidly decreasing

$$
\sup _{m, n}\left|t^{m} \partial^{n} \varphi(t)\right|<\infty \text {, for all } m, n>0 .
$$

Denote $\mathcal{S}_{+}(\mathbb{R})$ the subset of Schwartz functions having positive frequencies only $(\widehat{\varphi}(\omega)=0$ if $\omega \leq 0)$. For any such function, the Fourier transform is smoothly vanishing at zero or, what amounts to the same, all the moments cancel

$$
\widehat{\varphi}(\omega)=O\left(\omega^{n}\right) \Leftrightarrow \int d t t^{n} \varphi(t)=0, n \in \mathbb{N} .
$$

The reason for taking wavelets with no negative frequencies is that it considerably simplifies the computations and allows nice inversion formulae. Let us also introduce $S(\mathbb{H})$ the space of highly localized functions on the half plane, that is the functions $\mathcal{T}(b, a)$ satisfying

$$
\sup _{H}|\mathcal{T}(b, a)|\left(a+a^{-1}\right)^{m}(1+|b|)^{m}<\infty,
$$

for all $m>0$. Then the following holds true 
- If $g$ and $s$ are in $\mathcal{S}_{+}(\mathbb{R})$, then $\mathcal{W}_{g} s$ is in $\mathcal{S}(\mathbb{H})$.

- if $h$ is in $S_{+}(\mathbb{R})$ and $\mathcal{T}$ in $\mathcal{S}(\mathbb{H})$, then $\mathcal{M}_{h} \mathcal{T}$ is in $\mathcal{S}_{+}(\mathbb{R})$.

If furthermore the constant

$$
c_{g, h}=\int_{0}^{\infty} \frac{d \omega}{\omega} \widehat{h}(\omega) \overline{\widehat{g}}(\omega)
$$

is non zero, then we have the reconstruction formula

$$
c_{g, h}^{-1} \mathcal{M}_{h} \mathcal{W}_{g}=\mathbb{I}_{\mathcal{S}_{+}(R)},
$$

where $\mathbb{I}_{\mathcal{S}_{+}(\mathbb{R})}$ is the identity operator on $\mathcal{S}_{+}(\mathbb{R})$. Now, upon reconstructing with $g$ and analysing with $h$, we obtain the so called cross kernel equation, which relates the wavelet transforms with respect to different wavelets $g$ and $h$

$$
\mathcal{W}_{g} s(b, a)=\int_{\mathbb{H}} \frac{d a^{\prime}}{a^{\prime}} d b^{\prime} \frac{1}{a^{\prime}} P_{g \rightarrow h}\left(\frac{b-b^{\prime}}{a^{\prime}}, \frac{a^{\prime}}{a}\right) \mathcal{W}_{h} s\left(b^{\prime}, a^{\prime}\right),
$$

whith $P_{g \rightarrow h}(b, a)=c_{g, h}^{-1} \mathcal{W}_{h} g(b, a)$. If we introduce a (non commutative) convolution on $\mathcal{S}(\mathbb{H})$ by

$$
\mathcal{T}_{1} * \mathcal{T}_{2}(b, a)=\int_{I H} \frac{d a^{\prime}}{a^{\prime}} d b^{\prime} \frac{1}{a^{\prime}} \mathcal{T}_{1}\left(\frac{b-b^{\prime}}{a^{\prime}}, \frac{a}{a^{\prime}}\right) \mathcal{T}_{2}\left(b^{\prime}, a^{\prime}\right),
$$

then the above equation may be more simply rewritten as

$$
\mathcal{W}_{g} s(b, a)=P_{g \rightarrow h} * \mathcal{W}_{h} s(b, a),
$$

an important equation for the following. Thus, the passage from one wavelet to another in the half plane is done by convolution with a highly localized kernel. If $\mu$ is a Borel measure on $\mathbb{R}$, its wavelet transform with respect to a wavelet $g \in \mathcal{S}_{+}(\mathbb{R})$ is given by

$$
\mathcal{W}_{g} \mu(b, a)=\int d \mu(t) \frac{1}{a} \bar{g}\left(\frac{t-b}{a}\right)=\tilde{g}_{a} * \mu(b),
$$

and the cross kernel equation is still valid

$$
\mathcal{W}_{g} \mu(b, a)=P_{g \rightarrow h} * \mathcal{W}_{h} \mu(b, a) .
$$

Since we are interested in local properties, we will by now only consider finite Borel measures $\mu$ on $\mathbb{R}$. This in particular includes the case of functions in $L^{1}(\mathbb{R})$, which can be trivially identified with finite measures. Given some analysing wavelet $g \in \mathcal{S}_{+}(\mathbb{R})$ and some real $q \geq 1$, we define

$$
G_{g} \mu(a, q)=\left\|\mathcal{W}_{g} \mu(\cdot, a)\right\|_{q}^{q}=\int d b\left|\mathcal{W}_{g} \mu(b, a)\right|^{q} .
$$


The above quantity is finite since by Young's inequality (see the appendix)

$$
G_{g} \mu(a, q)=\left\|\tilde{g}_{a} * \mu\right\|_{q} \leq\|\mu\|_{1}\left\|\tilde{g}_{a}\right\|_{q}<\infty .
$$

At small scales, a scaling behavior of the form $G_{g} \mu(a, q) \sim a^{\kappa_{q}}$ can in general be observed giving rise to the definition of fractal dimensions $\kappa_{q}$. This approach has been developed in [2]. We propose to extend this definition by introducing a supplementary parameter. For $m \in \mathbb{R}$, we define the function

$$
\Gamma_{g} \mu(t, q, m)=\int_{t}^{1} \frac{d a}{a} a^{m} G_{g} \mu(a, q),
$$

and look at its small scale behavior $t \rightarrow 0$. Note that $\Gamma_{g} \mu(t, q, m)$ is a monotone function of $t$. Therefore, the limit exists, but may be infinite. In the opposite case when this limit is finite, we rather look at the rate of convergence by putting

$$
\Gamma_{g} \mu(t, q, m)=\int_{0}^{t} \frac{d a}{a} a^{m} G_{g} \mu(a, q) .
$$

To summarize, we have

$$
\Gamma_{g} \mu(t, q, m)=\min \left\{\int_{0}^{\mathrm{t}} \frac{\mathrm{da}}{\mathrm{a}} \mathrm{a}^{\mathrm{m}} \mathrm{G}_{\mathrm{g}} \mu(\mathrm{a}, \mathrm{q}), \int_{\mathrm{t}}^{1} \frac{\mathrm{da}}{\mathrm{a}} \mathrm{a}^{\mathrm{m}} \mathrm{G}_{\mathrm{g}} \mu(\mathrm{a}, \mathrm{q})\right\} .
$$

The generalized wavelet dimensions $\kappa_{q, m}^{ \pm}$are now defined by

$$
\kappa_{q, m}^{+}[\mu]=\limsup _{a \rightarrow 0} \frac{\log \Gamma_{g} \mu(a, q, m)}{\log a}, \kappa_{q, m}^{-}[\mu]=\liminf _{a \rightarrow 0} \frac{\log \Gamma_{g} \mu(a, q, m)}{\log a} .
$$

These are intrinsic dimensions of the measures $\mu$, as the following theorem shows.

Theorem 11.4.1 The generalized wavelet dimensions $\kappa_{q, m}^{ \pm}$are well defined in the sense that they do not depend on the analysing wavelet $g \in \mathcal{S}_{+}(\mathbb{R})$, provided $g \neq 0$.

The proof of this theorem is given in appendix.

\subsection{Time evolution and wavelet dimensions}

The generalized wavelet dimensions $\kappa_{q, m}^{ \pm}$can be related to the exponents $d_{m}^{ \pm}$introduced in section 11.1 in the following way. 
Theorem 11.5.1 Let $\mu$ be a finite Borel measure on $\mathbb{R}$ and let $h(t)=$ $|\widehat{\mu}(t)|^{2}$. Then we have for all integer $m \geq 0$

$$
d_{m}^{+}[h]=-\kappa_{2,-m}^{-}[\mu], \text { and } d_{m}^{-}[h]=-\kappa_{2,-m}^{+}[\mu] .
$$

The proof is also given in the appendix. An immediate corrolary is

$$
d_{l a c}[h]=-\lim _{m \rightarrow \infty} \frac{\kappa_{2,-m}^{+}[\mu]}{m} .
$$

This shows that the lacunary long time evolution generated by the Schrödinger equation is related to the generalized wavelet dimensions of the spectral measure of the Hamiltonian $H$.

We wish to conclude this chapter by some remarks on the bearing of wavelet dimensions in the above time evolution problem. The reader may reasonably ask why we introduced complicated fractal dimensions $\kappa_{q, m}^{ \pm}$and the non intuitive spectral measure $\mu$ to rewrite a quantity which is already physically interpretable, namely $d_{l a c}[h]$. The reason is the following. To form the spectral measure, we need three ingredients: the Hamiltonian itself, the initial state $\psi_{0}$ and the reference state $\phi$. Now these are time independent data. Thus, once the dynamics and the initial state of the system have been given, the equation (11.5.2) automatically provides the lacunarity dimension of $h(t)$. On the other hand, to compute directly the lacunarity dimension by means of the exponents $d_{m}^{-}[h]$ would require the full knowledge of $h(t)$ over a huge time span, possibly too long for measurements. Moreover, expressing the lacunarity dimension in terms of wavelet dimensions set up a precise correspondence between the long time evolution of the dynamical system and the fractal spectral properties of its generator (the Hamiltonian). The next natural question might be why we use wavelet dimensions and not "classical" fractal dimensions such as the correlation dimension, the box dimension, etc...The answer is simple: the usual fractal dimensions are not adapted to characterize signed or complex measures, whereas the wavelet dimensions are. For instance, the oscillating singularities appearing in "chirps" functions such as $\sin \left(|x|^{-\alpha}\right)$ are not detectable by means of the usual fractal dimensions whereas the wavelet dimensions can show them up. For positive measures, however, the wavelet dimensions can in some cases be related to better known fractal dimensions. In particular, it has been shown in [1] that for any finite positive measure $\mu$ we have 


$$
\kappa_{2}^{+}[\mu]=D^{+}[\mu] \text { and } \kappa_{2}^{-}[\mu]=D^{-}[\mu],
$$

where $D^{+}[\mu]$ and $D^{-}[\mu]$ are the upper respectively lower correlation $d i$ mension (see e.g [5]) of the measure $\mu$. Therefore, the lacunary dimension in the time evolution can be related to a classical fractal dimension of the spectral measure if this latter is positive. This is for example the case if the reference state coincides with the initial state, that is $\phi=\psi_{0}$ (see section 11.3 for notations). In the general case of complex spectral measures, the correlation dimension has to be replaced by the wavelet correlation dimension.

Acknowledgments. Many thanks to Hans van den Berg for his careful and patient reading and to the Laboratoire Geosciences-Rennes, where most of this work was done, for its warm hospitality.

\subsection{Appendix}

Proof of theorem 11.2.1 For the purpose of the proof, we introduce the notations

$$
H(T, m)=\int_{0}^{T} d t t^{m} h(t)
$$

and

$$
\eta(T, m)=\frac{\log H(T, m)}{\log T} .
$$

With this notation we have

$$
d_{m}^{+}[h]=\limsup _{T \rightarrow \infty} \eta(T, m), \text { and } d_{m}^{-}[h]=\liminf _{T \rightarrow \infty} \eta(T, m) .
$$

For fixed $m, H(T, m)$ is a non decreasing function of $T$ such that $H(T, m) \leq$ $T^{m} H(T, 0)$. Therefore,

$$
\begin{aligned}
& 0 \leq d_{m}^{+}[h] \leq d_{0}^{+}[h]+m, \\
& 0 \leq d_{m}^{-}[h] \leq d_{0}^{-}[h]+m .
\end{aligned}
$$

On the other hand $\eta(T, m)$ is, for fixed $T$, infinitely many times differentiable with respect to $m$. An elementary computation gives for $T>1$

$$
\frac{\partial \eta(T, m)}{\partial m} \geq 0, \frac{\partial^{2} \eta(T, m)}{\partial m^{2}} \geq 0
$$


that is $\eta(T, m)$ is a non decreasing convex function of $m$. Thus, for any $0 \leq \alpha \leq 1$, we have

$$
\eta(T, \alpha m) \leq \alpha \eta(T, m)+(1-\alpha) \eta(T, 0) .
$$

Now we use the inequalities

$$
\begin{aligned}
\lim \sup (f+g) & \leq \lim \sup f+\lim \sup g \\
\liminf (f+g) & \leq \lim \inf f+\lim \sup g
\end{aligned}
$$

which yield

$$
\begin{aligned}
& d_{\alpha m}^{+}[h] \leq \alpha d_{m}^{+}[h]+(1-\alpha) d_{0}^{+}[h] \\
& d_{\alpha m}^{-}[h] \leq \alpha d_{m}^{-}[h]+(1-\alpha) d_{0}^{+}[h],
\end{aligned}
$$

and thus

$$
\begin{aligned}
\frac{d_{\alpha m}^{+}[h]-d_{0}^{+}[h]}{\alpha m} \leq \frac{d_{m}^{+}[h]-d_{0}^{+}[h]}{m}, \\
\frac{d_{\alpha m}^{-}[h]-d_{0}^{+}[h]}{\alpha m} \leq \frac{d_{m}^{-}[h]-d_{0}^{+}[h]}{m} .
\end{aligned}
$$

Since any $m^{\prime}>m$ can be expressed as $m / \alpha$ with $0<\alpha<1$, this means that $\left(d_{m}^{+}[h]-d_{0}^{+}[h]\right) / m$ and $\left(d_{m}^{-}[h]-d_{0}^{+}[h]\right) / m$ are non decreasing functions of $m$. Now in view of 11.6.1 we have

$$
0 \leq \frac{d_{m}^{-}[h]-d_{0}^{+}[h]}{m} \leq \frac{d_{m}^{+}[h]-d_{0}^{+}[h]}{m} \leq 1 .
$$

It follows that the $\operatorname{limits}_{\lim } \lim _{m \rightarrow \infty} d_{m}^{ \pm}[h] / m$ exist and lie between zero and one. Finally, let us show that $\lim _{m \rightarrow \infty} d_{m}^{+}[h] / m$ is either zero or one. If $h$ is of compact support, this is evident because in this case $d_{m}^{ \pm}[h]=0$. So we may suppose that $h$ has unbounded support. Then look at

$$
\limsup _{T \rightarrow \infty} \frac{\log \left(\int_{T}^{T+1} d t h(t)\right)}{\log T} .
$$

If the above quantity is a finite constant, say $\alpha$, then we can find a sub-sequence $\left(T_{n}\right)$ and a constant $C>0$ for which

$$
\int_{T_{n}}^{T_{n}+1} d t h(t) \geq C T_{n}^{\alpha-1} .
$$

This gives

$$
\int_{1}^{T_{n}+1} t^{m} d t h(t) \geq \int_{T_{n}}^{T_{n}+1} t^{m} d t h(t) \geq C T_{n}^{m+\alpha-1},
$$


whence $d_{0}^{+}[h]+m \geq d_{m}^{+}[h] \geq m+\alpha-1$ and therefore $\lim _{m \rightarrow \infty} d_{m}^{+}[h] / m=$ 1. In the opposite case where

$$
\limsup _{T \rightarrow \infty} \frac{\log \left(\int_{T}^{T+1} d t h(t)\right)}{\log T}=-\infty
$$

it is not hard to see that $\int_{1}^{T} d t t^{m} h(t)$ is a convergent integral for all $m$ and therefore $d_{m}^{ \pm}[h]=0$. This proves the theorem.

Proof of example 2 Take some $\gamma^{\prime}$ with $1 \leq \gamma^{\prime} \leq \gamma$ and some integer $N$ and let us estimate

$$
\int_{0}^{b_{N}^{\gamma^{\prime}}} d t t^{m} h(t)=\sum_{n=0}^{\infty} \int_{0}^{b_{N}^{\gamma^{\prime}}} d t t^{m} \varphi\left(t-b_{n}\right) .
$$

To this end, let us look separately at each term appearing in the sum. While $n \leq N$, we have for any $\epsilon>0$

$$
\int_{0}^{b_{N}^{\gamma^{\prime}}} t^{m} \varphi\left(t-b_{n}\right) d t=\left\{\int_{0}^{b_{n}^{1-\epsilon}}+\int_{b_{n}^{1-\epsilon}}^{b_{n}^{1+\epsilon}}+\int_{b_{n}^{1+\epsilon}}^{b_{N}^{\gamma^{\prime}}}\right\} t^{m} \varphi\left(t-b_{n}\right) d t=I_{1}+I_{2}+I_{3} .
$$

Using the approximations $\varphi(t) \sim t^{-K}, t>>1$, we obtain the following estimates

$$
\begin{aligned}
& I_{1} \leq b_{n}^{(m-K+1)}, \\
& I_{3} \sim b_{N}^{\gamma^{\prime}(m-K+1)} .
\end{aligned}
$$

On the other hand we have

$$
\begin{aligned}
c b_{n}^{m(1-\epsilon)} & \leq b_{n}^{m(1-\epsilon)} \int_{b_{n}^{1-\epsilon}}^{b_{n}^{1+\epsilon}} \varphi\left(t-b_{n}\right) d t \\
& \leq I_{2} \\
& \leq b_{n}^{m(1+\epsilon)} \int_{b_{n}^{1-\epsilon}}^{b_{n}^{1+\epsilon}} \varphi\left(t-b_{n}\right) d t \leq C b_{n}^{m(1+\epsilon)}
\end{aligned}
$$

for some positive constants $c$ and $C$.

Thus, if we regroup the first $N$ terms of the sum, we obtain

$$
c^{\prime} b_{N}^{\rho\left(m, \gamma^{\prime}\right)(1-\epsilon)} \leq \sum_{n=0}^{N} \int_{0}^{b_{N}^{\gamma^{\prime}}} d t t^{m} \varphi\left(t-b_{n}\right) \leq C^{\prime} b_{N}^{\rho\left(m, \gamma^{\prime}\right)(1+\epsilon)}
$$

for some other positive constants $c^{\prime}$ and $C^{\prime}$, where $\rho\left(m, \gamma^{\prime}\right)=\max \left\{\gamma^{\prime}(m-K+\right.$ 
The contribution of the terms with $n>N$ is negligible because

$$
\begin{aligned}
\int_{0}^{b_{N}^{\gamma^{\prime}}} d t t^{m} \varphi\left(t-b_{n}\right) & \leq \int_{0}^{b_{N}^{\gamma^{\prime}}} d t t^{m} \varphi\left(b_{N}^{\gamma^{\prime}}-b_{n}\right) \\
& \sim b_{n}^{-K} b_{N}^{\gamma^{\prime}(m+1)}<<b_{N}^{\gamma^{\prime}(m-K+1)} .
\end{aligned}
$$

Therefore we have

$$
c^{\prime \prime} b_{N}^{\rho\left(m, \gamma^{\prime}\right)(1-\epsilon)} \leq \int_{0}^{b_{N}^{\gamma^{\prime}}} d t t^{m} h(t) \leq C^{\prime \prime} b_{N}^{\rho\left(m, \gamma^{\prime}\right)(1+\epsilon)}, N \rightarrow \infty
$$

with $c^{\prime \prime}, C^{\prime \prime}>0$. Since $\epsilon$ can be choosen arbitrarily small, it follows that $\limsup _{N \rightarrow \infty} \frac{\log \left(\int_{0}^{b_{N}^{\gamma^{\prime}}} d t t^{m} h(t)\right)}{\log b_{N}^{\gamma^{\prime}}}=\liminf _{N \rightarrow \infty} \frac{\log \left(\int_{0}^{b_{N}^{\gamma^{\prime}}} d t t^{m} h(t)\right)}{\log b_{N}^{\gamma^{\prime}}}=\frac{\rho\left(m, \gamma^{\prime}\right)}{\gamma^{\prime}}$.

This yields the following estimates for $d_{m}^{+}[h]$ and $d_{m}^{-}[h]$

$$
\begin{aligned}
& d_{m}^{+}[h] \geq \sup _{1 \leq \gamma^{\prime} \leq \gamma} \frac{\rho\left(m, \gamma^{\prime}\right)}{\gamma^{\prime}}, \\
& d_{m}^{-}[h] \leq \inf _{1 \leq \gamma^{\prime} \leq \gamma} \frac{\rho\left(m, \gamma^{\prime}\right)}{\gamma^{\prime}} .
\end{aligned}
$$

It turns out that the above inequalities are actually equalities. Indeed, fix some $\gamma^{\prime}$ and some $\epsilon>0$. For any $T>0$, we may find $N$ such that $b_{N}^{\gamma^{\prime}} \leq T<b_{N}^{\gamma^{\prime}(1+\epsilon)}$. Then

$\frac{\log b_{N}^{\gamma^{\prime}}}{\log T} \frac{\log \int_{0}^{b_{N}^{\gamma^{\prime}}} d t t^{m} h(t)}{\log b_{N}^{\gamma^{\prime}}} \leq \frac{\log \int_{0}^{T} d t t^{m} h(t)}{\log T} \leq \frac{\log b_{N}^{\gamma^{\prime}(1+\epsilon)} \log \int_{0}^{b_{N}^{\gamma^{\prime}(1+\epsilon)}} d t t^{m} h(t}{\log T} \frac{\log b_{N}^{\gamma^{\prime}(1+\epsilon)}}{\log }$

Taking successively the limit superior and inferior, this leads to

$$
\frac{\rho\left(m, \gamma^{\prime}\right)}{\gamma^{\prime}(1+\epsilon)} \leq d_{m}^{-}[h] \leq d_{m}^{+}[h] \leq \frac{\rho\left(m, \gamma^{\prime}\right)(1+\epsilon)}{\gamma^{\prime}} .
$$

Again we may choose $\epsilon$ arbitrarily small and since this holds for any $\gamma^{\prime}$ we have equalities in (11.6.4). Now we have to distinguish different regimes for $m$. If $m$ is small enough to have $m-K+1 \leq m / \gamma$, then $\rho\left(m, \gamma^{\prime}\right)=m$ for all $1 \leq \gamma^{\prime} \leq \gamma$. Consequently,

$$
d_{m}^{+}[h]=m, \text { and } d_{m}^{-}[h]=\frac{m}{\gamma} .
$$

If $m / \gamma \leq m-K+1$, then $\rho\left(m, \gamma^{\prime}\right) / \gamma^{\prime}=m / \gamma^{\prime}$ if $1 \leq \gamma^{\prime} \leq m /(m-K+1)$ and $\rho\left(m, \gamma^{\prime}\right) / \gamma^{\prime}=m-K+1$ if $m /(m-K+1) \leq \gamma^{\prime} \leq \gamma$. This yields

$$
d_{m}^{+}[h]=m, \text { and } d_{m}^{-}[h]=m-K+1 .
$$


The case of exponential localization can be obtained by letting $K \rightarrow \infty$, in which case (11.6.5) is verified for all $m$. This concludes the proof.

Proof of proposition 11.2.1 For the proof we need the following lemma, that we give without demonstration since it is well-known.

\section{Lemma 11.6.1}

$$
\begin{aligned}
\liminf _{t \rightarrow 0} \frac{\log s(t)}{\log t} & =\sup \left\{\gamma \in \mathbb{R} \mid s(t) \leq O\left(t^{\gamma}\right), t \rightarrow 0,\right\} \\
\limsup _{t \rightarrow 0} \frac{\log s(t)}{\log t} & =\sup \left\{\gamma \in \mathbb{R} \mid t^{\gamma} \leq O(s(t)), t \rightarrow 0 .\right\}
\end{aligned}
$$

We are now going to show that $d_{m}^{+}[h]=d_{m}^{-}[h]=\alpha+m$ for all $m \geq m_{0}$. First suppose $m_{0}=0$. Then, for all $m \geq 0$, we have $d_{m}^{-}[h] \leq d_{m}^{+}[h] \leq$ $m+\alpha$. Now let $\epsilon>0$. By lemma 11.6.1, we can find for all $\delta>0$ two positive constants $0<c<C$ such that

$$
c T^{\alpha-\delta} \leq \int_{1}^{T} d t h(t) \leq C T^{\alpha+\delta} .
$$

Rewriting this for $T^{1-\epsilon}$ in place of $T$ and opposing the sign gives

$$
-C T^{(\alpha+\delta)(1-\epsilon)} \leq-\int_{1}^{T^{1-\epsilon}} d t h(t) \leq-c T^{(\alpha-\delta)(1-\epsilon)}
$$

and adding line by line the last two inequalities yields

$$
c T^{\alpha-\delta}-C T^{(\alpha+\delta)(1-\epsilon)} \leq \int_{T^{1-\epsilon}}^{T} d t h(t) \leq C T^{\alpha+\delta} .
$$

Upon choosing $\delta$ small enough, we have $\alpha-\delta>(\alpha+\delta)(1-\epsilon)$ and

$$
c T^{\alpha-\delta} \leq \int_{T^{1-\epsilon}}^{T} d t h(t) \leq C T^{\alpha+\delta}
$$

Again by lemma 11.6.1, it follows that

$$
\limsup _{T \rightarrow \infty} \frac{\log \int_{T^{1-\epsilon}}^{T} d t t^{m} h(t)}{\log T}=\liminf _{T \rightarrow \infty} \frac{\log \int_{T^{1-\epsilon}}^{T} d t t^{m} h(t)}{\log T}=\alpha .
$$

Now, since $\int_{1}^{T} d t t^{m} h(t) \geq T^{m(1-\epsilon)} \int_{T^{1-\epsilon}}^{T} d t t^{m} h(t)$, this yields $m+\alpha \geq$ $d_{m}^{+}[h] \geq d_{m}^{-}[h] \geq \alpha+m(1-\epsilon)$. Since $\epsilon$ is arbitrary, this shows that $d_{m}^{+}[h]=d_{m}^{-}[h]=m+\alpha$, in which case the lacunarity dimension is one. If $m_{0} \neq 0$, we may apply the same reasoning to $t^{m_{0}} h(t)$ instead of $h(t)$ and the conclusion follows. 
Proof of theorem 11.4.1 Let us begin with some comments on the definition of the function $\Gamma_{g} \mu$. The rate of decay of the wavelet transform $\mathcal{W}_{g} \mu(b, a)$ as $a \rightarrow 0$ (resp. $a \rightarrow \infty$ ) reflects the behaviour of the Fourier transform $\hat{\mu}$ at $\infty$ (resp. 0). Precisely, we have

$$
\begin{aligned}
& \hat{\mu}(\omega) \leq O\left(\omega^{m}\right), \omega \rightarrow 0, \Rightarrow \mathcal{W}_{g} \mu(b, a) \leq O\left(a^{-m-1}\right), a \rightarrow \infty, \\
& \hat{\mu}(\omega) \leq O\left(\omega^{m}\right), \omega \rightarrow \infty, \Rightarrow \mathcal{W}_{g} \mu(b, a) \leq O\left(a^{m+1}\right), a \rightarrow 0
\end{aligned}
$$

uniformly in $b$. (This is a consequence of (11.4.1)). Thus, if $s$ is in $C^{\infty}(\mathbb{R}) \cap \mathcal{L}^{1}(\mathbb{R})$, then by $(11.6 .8), G_{g} s(a, q)=\left\|\mathcal{W}_{g} s(\cdot, a)\right\|_{q}^{q}$ is rapidly decaying at small scales. It follows that $\mu$ and $\mu+s$ have the same wavelet-dimensions $\kappa^{ \pm}(q, m)$. Hence, if we define $\langle\mu\rangle$ the class of equivalence of $\mu$ modulo smooth functions ( that is $\left\langle\mu^{\prime}\right\rangle=\langle\mu\rangle$ if $\mu^{\prime}-\mu$ can be identified to a $C^{\infty}$ function), then two measures belonging to the same class $\langle\mu\rangle$ have the same wavelet dimensions. Now, for a given measure $\mu$, we always can find $\mu^{\prime}$ in $\langle\mu\rangle$ whose Fourier transform is flat around $O$. It suffices to takes $\mu^{\prime}=\mu-\phi * \mu$ with $\phi \in \mathcal{S}(\mathbb{R})$ and $\hat{\phi}(\omega)=1+O\left(\omega^{m}\right), \omega \rightarrow 0$, for all $m$. Therefore, we may assume that condition (11.6.8) holds when we compute the wavelet dimensions. In that case, $G_{g} \mu(a, q)$ is rapidly decreasing at large scales and we may thus replace $\int_{t}^{1}$ by $\int_{t}^{\infty}$ in the definition of $\Gamma_{g} \mu(t, q, m)$, that is we may set

$$
\Gamma_{g} \mu(t, q, m)=\int_{t}^{\infty} \frac{d a}{a} a^{m} G_{g} \mu(a, q) .
$$

With this remark in mind, we can begin the proof. Take $g$ and $h$ two analysing wavelets in $\mathcal{S}_{+}(\mathbb{R})$. Let us compare $\Gamma_{g} \mu(t, q, m)$ and $\Gamma_{h} \mu(t, q, m)$ as $t \rightarrow 0$. From equation (11.4.5) it follows that with

$$
K_{a^{\prime}, a}(b)=\frac{1}{a^{\prime}} P_{g \rightarrow h}\left(\frac{b}{a^{\prime}}, \frac{a}{a^{\prime}}\right)
$$

the passage from $\mathcal{W}_{g} \mu$ to $\mathcal{W}_{h} \mu$ reads

$$
\mathcal{W}_{h} \mu(\cdot, a)=\int_{0}^{\infty} \frac{d a^{\prime}}{a^{\prime}} K_{a^{\prime}, a} * \mathcal{W}_{g} \mu\left(\cdot, a^{\prime}\right)
$$

However we have to make sure that $K_{a^{\prime}, a}$ is well defined. The only possible obstruction to this is the constant $c_{g, h}$ as defined in (11.4.2) which may vanish. (Note that it is never $\infty$ for $g, h \in \mathcal{S}_{+}(\mathbb{R})$.) However it cannot vanish for all the dilated and translated versions $g_{\beta, \alpha}=$ 
$\alpha^{-1} g([\cdot-\beta] / \alpha)$ of $g$ since this would merely mean that the wavelet transform of $h$ with respect to $g$ vanishes, which is impossible for $h \neq 0$. Now replacing $g$ by one of its dilated and translated versions $g_{\beta, \alpha}$ amounts to replace $\mathcal{W}_{g} \mu(b, a)$ by

$$
\mathcal{W}_{g_{\beta, \alpha}} \mu(b, a)=\frac{1}{\alpha} \mathcal{W}_{g} \mu\left(\frac{b-\beta}{\alpha}, \frac{a}{\alpha}\right)
$$

and therefore the dimensions computed with $g_{\beta, \alpha}$ instead of $g$ are the same. We therefore may suppose that $c_{g, h} \neq 0$.

Now we have

$$
\begin{aligned}
\left\|\mathcal{W}_{h} \mu(\cdot, a)\right\|_{q} & =\left(\int d b\left|\mathcal{W}_{h} \mu(b, a)\right|^{q}\right)^{1 / q} \\
& \leq\left\{\int d b\left(\int_{0}^{\infty} \frac{d a^{\prime}}{a^{\prime}}\left|K_{a^{\prime}, a} * \mathcal{W}_{g} \mu\left(\cdot, a^{\prime}\right)(b)\right|\right)^{q}\right\}^{1 / q} \\
& \leq \int_{0}^{\infty} \frac{d a^{\prime}}{a^{\prime}}\left\{\int d b\left|K_{a^{\prime}, a} * \mathcal{W}_{g} \mu\left(\cdot, a^{\prime}\right)(b)\right|^{q}\right\}^{1 / q} \text { by Minkowski's } \\
& =\int_{0}^{\infty} \frac{d a^{\prime}}{a^{\prime}}\left\|K_{a^{\prime}, a} * \mathcal{W}_{g} \mu\left(\cdot, a^{\prime}\right)\right\|_{q} \\
& \leq \int_{0}^{\infty} \frac{d a^{\prime}}{a^{\prime}}\left\|K_{a^{\prime}, a}\right\|_{1}\left\|\mathcal{W}_{g} \mu\left(\cdot, a^{\prime}\right)\right\|_{q} \text { by Young's inequality }
\end{aligned}
$$

On the other hand,

$$
\left\|K_{a^{\prime}, a}\right\|_{1}=\int_{-\infty}^{+\infty} d b \frac{1}{a^{\prime}}\left|P_{g \rightarrow h}\left(\frac{b}{a^{\prime}}, \frac{a}{a^{\prime}}\right)\right| H\left(a / a^{\prime}\right),
$$

with

$$
H(a)=\int_{-\infty}^{+\infty} d b\left|P_{g \rightarrow h}(b, a)\right|
$$

This is a nonnegative function that is rapidly decaying as $a+1 / a$ gets large. Now set

$$
\Lambda=\int_{0}^{\infty} \frac{d a^{\prime}}{a^{\prime}} H\left(a / a^{\prime}\right)
$$

which is a finite constant thanks to the high localization of $H$, and

$$
d \nu\left(a^{\prime}\right)=\Lambda^{-1} \frac{d a^{\prime}}{a^{\prime}} H\left(a / a^{\prime}\right),
$$

which is a probabilty measure. Then, using Jensen's inequality, we obtain

$$
\left\|\mathcal{W}_{h} \mu(\cdot, a)\right\|_{q}^{q}=\Lambda^{q}\left(\int_{0}^{\infty} d \nu\left(a^{\prime}\right)\left\|\mathcal{W}_{g} \mu\left(\cdot, a^{\prime}\right)\right\|_{q}\right)^{q}
$$




$$
\begin{aligned}
& \leq \Lambda^{q} \int_{0}^{\infty} d \nu\left(a^{\prime}\right)\left\|\mathcal{W}_{g} \mu\left(\cdot, a^{\prime}\right)\right\|_{q}^{q} \\
& =\Lambda^{q-1} \int_{0}^{\infty} \frac{d a^{\prime}}{a^{\prime}} H\left(a / a^{\prime}\right)\left\|\mathcal{W}_{g} \mu\left(\cdot, a^{\prime}\right)\right\|_{q}^{q} .
\end{aligned}
$$

Now suppose that we are in the case

$$
\lim _{t \rightarrow 0} \int_{t}^{1} \frac{d a}{a} a^{m} G_{g} \mu(a, q)=\infty .
$$

Then, as was explained in the last remark, we may compute the wavelet dimension with

$$
\Gamma_{g} \mu(t, q, m)=\int_{t}^{\infty} \frac{d a}{a} a^{m} G_{g} \mu(a, q) .
$$

With this assumption, (11.6.9) yields

$$
\begin{aligned}
\Gamma_{h} \mu(t, q, m) & =\int_{t}^{\infty} \frac{d a}{a} a^{m}\left\|\mathcal{W}_{h} \mu(\cdot, a)\right\|_{q}^{q} \\
& \leq O(1) \int_{t}^{\infty} \frac{d a}{a} a^{m} \int_{0}^{\infty} \frac{d a^{\prime}}{a^{\prime}} H\left(a / a^{\prime}\right)\left\|\mathcal{W}_{g} \mu\left(\cdot, a^{\prime}\right)\right\|_{q}^{q} \\
& =O(1) \int_{0}^{\infty} \frac{d a^{\prime}}{a^{\prime}} H\left(1 / a^{\prime}\right) \int_{t}^{\infty} \frac{d a}{a} a^{m}\left\|\mathcal{W}_{g} \mu\left(\cdot, a a^{\prime}\right)\right\|_{q}^{q} \\
& =O(1) \int_{0}^{\infty} \frac{d a^{\prime}}{a^{\prime}} a^{\prime-m} H\left(1 / a^{\prime}\right) \int_{t a^{\prime}}^{\infty} \frac{d a}{a} a^{m}\left\|\mathcal{W}_{g} \mu(\cdot, a)\right\|_{q}^{q} . \\
& =O(1) \int_{0}^{\infty} \frac{d a^{\prime}}{a^{\prime}} H\left(1 / a^{\prime}\right) \Gamma_{g} \mu\left(t a^{\prime}, q, m\right) \\
& =O(1) \int_{0}^{\infty} \frac{d a^{\prime}}{a^{\prime}} H\left(t / a^{\prime}\right) \Gamma_{g} \mu\left(a^{\prime}, q, m\right),
\end{aligned}
$$

that is

$$
\Gamma_{h} \mu(t, q, m) \leq O(1) \int_{0}^{\infty} \frac{d a}{a} H(t / a) \Gamma_{g} \mu(a, q, m) .
$$

As can be easily checked, the same relation holds in the alternative case

$$
\Gamma_{g} \mu(t, q, m)=\int_{0}^{t} \frac{d a}{a} a^{m} G_{g} \mu(a, q) .
$$

Since $g$ and $h$ can be exchanged in the above inequality, it follows that $\dagger$

$$
\Gamma_{h} \mu(t, q, m) \sim \int_{0}^{\infty} \frac{d a}{a} H(t / a) \Gamma_{g} \mu(a, q, m) .
$$

$\dagger$ The notation $f \sim g$ means $C^{-1} f(x) \leq g(x) \leq C f(x)$ for some constant $C>0$ 
Note that the integral on the right-hand side is always finite because $\Gamma_{g} \mu(a, q, m)$ is of at most polynomial grothw in $a+1 / a$ whereas $H$ is rapidly decreasing in $a+1 / a$.

Now suppose that $\Gamma_{g} \mu(t, q, m) \leq O\left(t^{\gamma}\right), t \rightarrow 0$, for some $\gamma$. Then by (11.6.10), we have

$$
\Gamma_{h} \mu(t, q, m) \leq O\left(t^{\gamma}\right) \int_{0}^{\infty} \frac{d a}{a} H(1 / a) a^{\gamma} \leq O\left(t^{\gamma}\right), t \rightarrow 0 .
$$

Since $g$ and $h$ can be exchanged in (11.6.10), it follows that, for all $\gamma$

$$
\Gamma_{h} \mu(t, q, m) \leq O\left(t^{\gamma}\right) \Leftrightarrow \Gamma_{g} \mu(t, q, m) \leq O\left(t^{\gamma}\right), t \rightarrow 0 .
$$

Conversely, suppose that $\Gamma_{g} \mu(t, q, m) \geq C t^{\gamma}, 0<t<1$ for some constant $C>0$. Things are here slightly more complicated. Pick some $\epsilon$, $0<\epsilon<1$, and keep it fixed. For $0<t<1$ we split the integral of (11.6.10) into three parts

$\Gamma_{h} \mu(t, q, m)=\left\{\int_{0}^{t^{1+\epsilon}}+\int_{t^{1+\epsilon}}^{t^{1-\epsilon}}+\int_{t^{1-\epsilon}}^{\infty}\right\} \frac{d a}{a} H(t / a) \Gamma_{g} \mu(a, q, m)=X_{1}+X_{2}+X_{3}$

In the last term we may estimate $\Gamma_{g} \mu(t, q, m) \leq O(1)$ and thus

$$
X_{3} \leq O(1) \int_{1 / t^{\epsilon}}^{\infty} \frac{d a}{a} H(1 / a)
$$

Since $H(t)$ is arbitrarily well polynomially localized it follows that $X_{3}=$ $O\left(t^{n}\right)$ for all $n>0$.

In $X_{1}$ we may estimate $\Gamma_{g} \mu(t, q, m) \leq t^{-p}$ for some $p$ because $\Gamma_{g} \mu(t, q, m)$ is rapidly decreasing in $t+1 / t$ and thus

$$
X_{1} \leq O(1) t^{-p} \int_{0}^{t^{\epsilon}} \frac{d a}{a} H(1 / a) a^{-p} .
$$

Since $H$ is arbitrary well polynomially localized the integral is rapidly decaying and thus again $X_{1}=O\left(t^{n}\right)$ for all $n>0$.

The remaining contribution is the middle term $X_{2}$. If $\Gamma_{g} \mu(t, q, m)$ is non decreasing, then

$$
\begin{aligned}
X_{2} & =\int_{t^{\epsilon}}^{t^{-\epsilon}} \frac{d a}{a} \Gamma_{g} \mu(a t, q, m) H(1 / a) \\
& \geq \int_{t^{\epsilon}}^{1} \frac{d a}{a} H(1 / a) \Gamma_{g} \mu(a t, q, m) \\
& \geq \Gamma_{g} \mu\left(t^{1+\epsilon}, q, m\right) \int_{t^{1+\epsilon}}^{1} \frac{d a}{a} H(1 / a) \\
& \geq \Gamma_{g} \mu\left(t^{1+\epsilon}, q, m\right) \int_{0}^{1} \frac{d a}{a} H(1 / a) \\
& \geq C^{\prime} t^{\gamma(1+\epsilon)} .
\end{aligned}
$$


If $\left.\Gamma_{g} \mu(t, q, m) t\right)$ is non increasing, then

$$
\begin{aligned}
X_{2} & \geq \int_{1}^{t^{-\epsilon}} \frac{d a}{a} H(1 / a) \Gamma_{g} \mu(a t, q, m) \\
& \geq \Gamma_{g} \mu\left(t^{1+\epsilon}, q, m\right) \int_{1}^{t^{-\epsilon}} \frac{d a}{a} H(1 / a) \\
& \geq \Gamma_{g} \mu\left(t^{1+\epsilon}, q, m\right) \int_{1}^{\infty} \frac{d a}{a} H(1 / a) \\
& \geq C^{\prime} t^{\gamma(1+\epsilon)} .
\end{aligned}
$$

Thus, we have for all $\gamma$ and all $\epsilon>0$

$$
C t^{\gamma} \leq \Gamma_{g} \mu(t, q, m) \Rightarrow t^{\gamma+\epsilon} \leq C^{\prime} \Gamma_{h} \mu(t, q, m),
$$

and also, since $g$ and $h$ can be interchanged

$$
C t^{\gamma} \leq \Gamma_{h} \mu(t, q, m) \Rightarrow t^{\gamma+\epsilon} \leq C^{\prime} \Gamma_{g} \mu(t, q, m),
$$

Once we have proven (11.6.11), (11.6.12) and (11.6.13), the conclusion follows from lemma (11.6.1).

Proof of theorem 11.5.1 Take some wavelet $g \in \mathcal{S}_{+}(\mathbb{R})$ such that $\widehat{g}$ is compactly supported. Again we may suppose in addition that $\widehat{\mu}(\omega)=$ $O\left(\omega^{m}\right)$ for all $m$, whence $\mathcal{W}_{g} \mu$ is rapidly decaying at large scale. A direct application of Parsevals equation gives

$$
\int_{-\infty}^{+\infty} d b\left|\mathcal{W}_{g} \mu(b, a)\right|^{2}=\int_{0}^{\infty} d \omega|\widehat{g}(a \omega)|^{2}|\widehat{\mu}(\omega)|^{2},
$$

and thus, by a simple exchange of integration

$$
\int_{I H} \frac{d a}{a} d b a^{-m}\left|\mathcal{W}_{g} \mu(b, a)\right|^{2}=\int_{0}^{\infty} \frac{d a}{a} a^{-m}|\widehat{g}(a)|^{2} \int_{0}^{\infty} d \omega \omega^{m}|\hat{\mu}(\omega)|^{2} .
$$

The first integral on the right-hand side is a finite constant, due to the high localization of $\hat{g}$. The second integral may be finite or not. First suppose it is infinite. Then we have

$$
\Gamma_{g} \mu\left(T^{-1}, 2,-m\right)=\int_{T^{-1}}^{\infty} \frac{d a}{a} a^{-m} \int_{-\infty}^{+\infty} d b\left|\mathcal{W}_{g} \mu(b, a)\right|^{2}
$$

By equation (11.6.14), this can be rewritten as

$$
\Gamma_{g} \mu\left(T^{-1}, 2,-m\right)=\int_{0}^{\infty} d \omega \omega^{m} H(\omega / T)|\widehat{\mu}(\omega)|^{2},
$$

with

$$
H(t)=\int_{t}^{\infty} \frac{d a}{a} a^{-m}|\widehat{g}(a)|^{2} .
$$


Since $H$ is non negative and of compact support (since $\widehat{g}$ is), we can find numbers $\lambda>0$ and $\Lambda>0$ such that

$$
\lambda \chi_{[0, \lambda]}(\omega) \leq H(\omega) \leq \Lambda \chi_{[0, \Lambda]}(\omega)
$$

where $\chi_{I}$ is the characteristic function of $I$. Therefore

$$
\lambda \int_{0}^{\lambda T} d \omega \omega^{m}|\widehat{\mu}(\omega)|^{2} \leq \Gamma_{g} \mu\left(T^{-1}, 2,-m\right) \leq \Lambda \int_{0}^{\Lambda T} d \omega \omega^{m}|\widehat{\mu}(\omega)|^{2},
$$

and it follows that

$$
\begin{aligned}
d_{m}^{+}\left[|\widehat{\mu}|^{2}\right] & =\limsup _{T \rightarrow \infty} \frac{\log \left(\int_{0}^{\lambda T} d \omega \omega^{m}|\widehat{\mu}(\omega)|^{2}\right)}{\log T} \\
& =\limsup _{T \rightarrow \infty} \frac{\log \Gamma_{g} \mu\left(T^{-1}, 2,-m\right)}{\log T} \\
& =-\kappa_{2,-m}^{-}[\mu],
\end{aligned}
$$

and

$$
\begin{aligned}
d_{m}^{-}\left[|\widehat{\mu}|^{2}\right] & =\liminf _{T \rightarrow \infty} \frac{\log \left(\int_{0}^{\lambda T} d \omega \omega^{m}|\widehat{\mu}(\omega)|^{2}\right)}{\log T} \\
& =\liminf _{T \rightarrow \infty} \frac{\log \Gamma_{g} \mu\left(T^{-1}, 2,-m\right)}{\log T} \\
& =-\kappa_{2,-m}^{+}[\mu],
\end{aligned}
$$

The proof for the case

$$
\int d \omega \omega^{m}|\widehat{\mu}(\omega)|^{2}<\infty
$$

is similar, we only have to use

$$
\Gamma_{g}\left(T^{-1}, 2,-m\right)=\int_{0}^{T^{-1}} \frac{d a}{a} a^{-m} \int_{-\infty}^{+\infty} d b\left|\mathcal{W}_{g} \mu(b, a)\right|^{2}
$$

and to adapt the limits of integration accordingly. This concludes the proof.

Some useful inequalities As usual $L^{p}(\mathbb{R})$ is the space of measurable functions $f$ for which

$$
\|f\|_{p}=\left(\int d t|f(t)|^{p}\right)^{\frac{1}{p}}<\infty .
$$


Hölder's inequality. If $f \in L^{p}(\mathbb{R})$ and $g \in L^{q}(\mathbb{R})$ with $1 / p+1 / q=1 / r$, then we have

$$
\|f g\|_{r} \leq\|f\|_{p}\|g\|_{q}
$$

Minkowsky's inequality. For any $p \geq 1$ we have

$$
\|f+g\|_{p} \leq\|f\|_{p}+\|g\|_{p} .
$$

Integral Minkowsky's inequality. If $f(x, y) \in L^{p}(\mathbb{R}) \times L^{p}(\mathbb{R})$ with $p \geq 1$,

$$
\left\{\int d y\left(\left|\int d x f(x, y)\right|^{p}\right)\right\}^{1 / p} \leq \int d x\left(\int d y|f(x, y)|^{p}\right)^{1 / p} .
$$

Young's inequality. If $f \in L^{p}(\mathbb{R})$ and $g \in L^{q}(\mathbb{R})$ with $1 / p+1 / q=$ $1+1 / r$, then

$$
\|f * g\|_{r} \leq\|f\|_{p}\|g\|_{q}
$$

Jensen's inequality. If $\mu$ is a probability measure and $\varphi$ a convex function, then we have

$$
\varphi\left(\int d \mu(t) f(t)\right) \leq \int d \mu(t) \varphi \circ f(t) .
$$




\section{Bibliography}

[1] Guerin C.A. and Holschneider M. On equivalent definitions of the correlation dimension for a probability measure, Journ. Stat. Phys, 86(3/4): 707-720, (1997).

[2] Holschneider M. Fractal wavelet dimension and localization, Comm. Math. Phys., 160: 457-473, (1994).

[3] Holschneider M. Wavelets, an Analysis Tool, Oxford University Press, 1995.

[4] Ketzmerick R., Petschel G. and Geisel T. Slow decay of temporal correlations in quantum systems with cantor spectra, Phys. Rev. Lett., 69(5): 695-698, 1992.

[5] Pesin Ya. P. On rigorous mathematical definition of the correlation dimension and generalized spectrum for dimension, Journ. Stat. Phys., 71(3/4): 529-547, 1993.

[6] Reed M. and Simon B. Functional Analysis, Academic Press, New York, 1980. 


\section{Index}

correlation dimension, 17

cross-kernel, 14

evolution operator, 11

hamiltonian system, 3

intermittency, 4

lacunarity dimension, 8

Poincaré recurrence theorem, 4

reconstruction formula, 14

spectral measure, 11

survival probability, 11

wavelet, 12

analysis, 13

correlation dimension, 12

generalized dimensions15

synthesis, 13

transform, 12 